\title{
Association between frequency of telephonic contact and clinical testing for a large, geographically diverse diabetes disease management population
}

\author{
Carter R. Coberley \\ Healthways, Inc., Nashville \\ Matthew McGinnis \\ Healthways, Inc., Nashville \\ Patty M. Orr \\ Healthways, Inc., Nashville \\ Baldbie/ \$hiGaberdelditional works at: https://jdc.jefferson.edu/healthpolicyfaculty \\ 'd-althways, Inc., Nashville \\ Part of the Health Services Research Commons \\ Adantighopow how access to this document benefits you \\ Healthways, Inc., Nashville
}

\section{Recommended Citation}

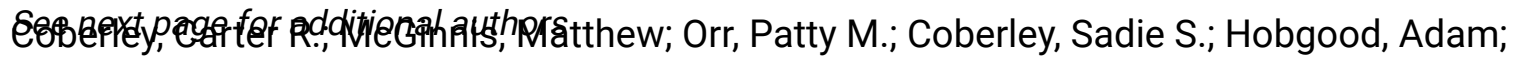
Hamar, Brent; Gandy, Bill; Pope, James; Hudson, Laurel; Hara, Pam; Shurney, Dexter; Clarke, Janice L.; Crawford, Albert ; and Goldfarb, Neil I., "Association between frequency of telephonic contact and clinical testing for a large, geographically diverse diabetes disease management population" (2007). College of Population Health Faculty Papers. Paper 33.

https://jdc.jefferson.edu/healthpolicyfaculty/33

This Article is brought to you for free and open access by the Jefferson Digital Commons. The Jefferson Digital Commons is a service of Thomas Jefferson University's Center for Teaching and Learning (CTL). The Commons is a showcase for Jefferson books and journals, peer-reviewed scholarly publications, unique historical collections from the University archives, and teaching tools. The Jefferson Digital Commons allows researchers and interested readers anywhere in the world to learn about and keep up to date with Jefferson scholarship. This article has been accepted for inclusion in College of Population Health Faculty Papers by an authorized administrator of the Jefferson Digital Commons. For more information, please contact: JeffersonDigitalCommons@jefferson.edu. 
Authors

Carter R. Coberley, Matthew McGinnis, Patty M. Orr, Sadie S. Coberley, Adam Hobgood, Brent Hamar, Bill Gandy, James Pope, Laurel Hudson, Pam Hara, Dexter Shurney, Janice L. Clarke, Albert Crawford, and Neil I. Goldfarb 


\title{
Association between Frequency of Telephonic Contact and Clinical Testing for a Large, Geographically Diverse Diabetes Disease Management Population
}

\author{
CARTER R. COBERLEY, Ph.D., ${ }^{1}$ MATTHEW McGINNIS, B.S., ${ }^{1}$ \\ PATTY M. ORR, M.S.N., Ed.D., R.N., ${ }^{1}$ SADIE S. COBERLEY, Ph.D., ${ }^{1}$ \\ ADAM HOBGOOD, M.S., ${ }^{1}$ BRENT HAMAR, D.D.S., M.P.H., ${ }^{1}$ BILL GANDY, Ed.D., ${ }^{1}$ \\ JAMES POPE, M.D., ${ }^{1}$ LAUREL HUDSON, R.N., M.S.N., ${ }^{1}$ PAM HARA, R.N., M.B.A., ${ }^{1}$ \\ DEXTER SHURNEY, M.D., M.B.A., M.P.H., ${ }^{1}$ JANICE L. CLARKE, R.N., ${ }^{2}$ \\ ALBERT CRAWFORD, Ph.D., M.B.A., M.S.I.S., ${ }^{2}$ and NEIL I. GOLDFARB, B.A. ${ }^{2}$
}

\begin{abstract}
Diabetes disease management (DM) programs strive to promote healthy behaviors, including obtaining hemoglobin A1c (A1c) and low-density lipoprotein (LDL) tests as part of standards of care. The purpose of this study was to examine the relationship between frequency of telephonic contact and A1c and LDL testing rates. A total of 245,668 members continuously enrolled in diabetes DM programs were evaluated for performance of an A1c or LDL test during their first 12 months in the programs. The association between the number of calls a member received and clinical testing rates was examined. Members who received four calls demonstrated a $24.1 \%$ and $21.5 \%$ relative increase in A1c and LDL testing rates, respectively, compared to members who received DM mailings alone. Response to the telephonic intervention as part of the diabetes DM programs was influenced by member characteristics including gender, age, and disease burden. For example, females who received four calls achieved a $27.7 \%$ and $23.6 \%$ increase in A1c and LDL testing, respectively, compared to females who received mailings alone; by comparison, males who were called achieved $21.2 \%$ and $19.9 \%$ relative increase in A1c and LDL testing, respectively, compared to those who received mailings alone. This study demonstrates a positive association between frequency of telephonic contact and increased performance of an A1c or LDL test in a large, diverse diabetes population participating in DM programs. The impact of member characteristics on the responsiveness to these programs provides DM program designers with knowledge for developing strategies to promote healthy behaviors and improve diabetes outcomes. (Disease Management 2007;10:101-109)
\end{abstract}

\section{INTRODUCTION}

$\mathbf{N}$ ATIONAL STATISTICS for 2005 report that 20.8 million people $-7 \%$ of the US popula- tion-have diabetes. Total costs attributed to the condition topped $\$ 132$ billion in $2002 .^{1}$ There is abundant evidence that improving glycemic control creates benefits for persons

\footnotetext{
${ }^{1}$ Healthways, Inc., Nashville, Tennessee.

${ }^{2}$ Department of Health Policy, Jefferson Medical College, Philadelphia, Pennsylvania.
} 
with diabetes by decreasing short- and longterm diabetes complications and lowering diabetes-related costs. ${ }^{2-5}$ In general, for every percentage point drop in hemoglobin A1c (A1c; eg, from $8 \%$ to $7 \%$ ), there is a corresponding reduction of $40 \%$ in the risk of microvascular complications (eg, retinopathy, kidney failure, peripheral neuropathy). ${ }^{2}$

Type 2 diabetes is associated with a two- to fourfold excess risk of cardiovascular disease (CVD). This is of particular importance as CVD is the major cause of death in patients with type 2 diabetes. ${ }^{6}$ Numerous studies have identified cholesterol (ie, low-density lipoprotein [LDL], high-density lipoprotein [HDL], and triglycerides) as an independent predictor of CVD. 7,8 These and other studies suggest that improved cholesterol control can reduce cardiovascular complications $20 \%-50 \%{ }^{9}$

Because of the significant risks of uncontrolled blood sugar and lipids for people with diabetes, the American Diabetes Association (ADA) calls for A1c testing at least twice a year for patients meeting glycemic control treatment goals, and quarterly testing for patients not meeting these goals. The ADA also calls for lipid testing at least annually. ${ }^{10}$ Adherence to these guidelines is critical for appropriate disease management (DM) and to reduce the risk of diabetes exacerbations. Studies have demonstrated that patients who obtain regular clinical tests are more likely to improve poor glycemic levels and achieve metabolic control. ${ }^{11-13}$

A key focus of diabetes DM programs is motivating members toward healthy behaviors, including obtaining recommended clinical tests. Results of a recent Healthways study demonstrated an association between patient participation in their diabetes DM programs and a measurable improvement in A1c testing. ${ }^{14}$ Other studies in the DM literature also speak to the positive impact of diabetes DM on frequency of testing and promotion of healthy behaviors directed toward improved control of diabetes. ${ }^{15-17}$ Less is known about which specific components of DM programs drive the improved outcomes. For instance, telephonic intervention through clinician-staffed call centers is believed to be an important component of the overall strategy to educate members, en- courage self-management, and identify opportunities for improving care delivery and coordination. However, there is little published information on the impact of telephonic intervention on driving large, diverse diabetes populations toward achieving the overall goals of diabetes DM.

The purpose of this retrospective observational study was to determine the relationship between frequency of telephonic contact and performance of recommended A1c and LDL testing in a large, multiregional, multi-health plan diabetes DM program. Several unique features of this study demonstrate the significance of these findings to other DM programs. Broad applicability and large population sample size were priorities in designing the study, ensuring that the approach would be useful in future analyses of DM programs targeting other chronic conditions. Further, members were grouped together based on the amount of time that they were exposed to DM as opposed to evaluating different DM calendar or contractual years. This approach allowed a more consistent evaluation of program effect based on length of participation in the program. In addition, this study sought to identify variations in clinical testing rates with respect to age, gender, and/or burden of disease in order to better understand how population diversity may impact healthy behaviors.

\section{METHODS}

\section{Healthways diabetes disease management program overview}

The diabetes DM programs were designed to work collaboratively and proactively with persons with diabetes, via telephone and mail, to ensure that each member understands his or her condition and the reasons for adhering to standards of care and treatment regimens. Participation in the programs is an "opt-out" or engagement process whereby health plan members with diabetes are automatically enrolled in the DM programs with the right to decline participation at any time. Telephone calls on behalf of members under the age of 18 are 
directed to a parent or legal guardian unless the parent gives permission for the child to receive the call directly. Similarly, elderly or disabled members may give approval for a relative or caregiver to receive calls on their behalf.

The call centers in this study were staffed with highly experienced clinicians (ie, registered nurses, registered dieticians) who initiated and maintained telephonic relationships with DM program members. Telephonic member support included several types of phone calls including introductory welcome calls, regularly scheduled care calls based on level of disease severity, and reminder calls regarding standards of care. Mail interventions included quarterly newsletters, reminder mailings, and disease-specific educational materials. Following introduction letters to the members and their corresponding physicians, welcome packets containing a detailed explanation of the program were mailed to members. Additional educational materials were mailed to members as needed.

\section{Study population}

A standardized outcomes data set was developed from a multiregional population of diabetes DM program members. The study period was defined as January 1, 2001 through December 31, 2004. This data set encompassed 245,668 unique members with diabetes from 25 different health plans across the United States.

A key design feature was standardization of the data, that is, replacing the health plan-specific contract or business logic with uniform criteria for eligibility, costs, and metrics. Using Structured Query Language (SQL), a large standardized Oracle 9i database was queried to identify members who were continuously enrolled in their first 12 months of the diabetes DM programs. For the purpose of this study, members were grouped together based on the number of months they participated in DM programs, irrespective of the contract year. This grouping was performed to account for the fact that in any given DM program contract year, members will have participated in the programs for varying periods of time. By grouping members in this way, it is possible to more uniformly account for varying lengths of participation in the DM programs.

\section{Clinical testing}

Two clinical process metrics-A1c and LDL testing-were examined to measure adherence to standards of care relative to the number of telephonic interventions received by a member during their first 12 months in the program. The primary outcome variables were whether members had an A1c or an LDL test during the same 12-month period. In order to be as conservative as possible, only administrative claims data were evaluated for A1c or LDL testing; self-reported data were not used in this study.

\section{Response to telephonic intervention}

Health plans selected which particular DM intervention packages their members received, which determines the level of telephonic contact. For instance, one DM intervention package only targeted members with the greatest disease burden (ie, top $20 \%$ of the population) to receive calls, while the remaining members only received one welcome call. In contrast, another DM intervention package targeted all members to receive four standard of care calls per year. The results of this study represent an aggregate of all the DM interventions, contributing to even greater population diversity.

The A1c and LDL testing rates of members in response to telephonic interventions during their first year in the DM programs were assessed. Members were grouped into cohorts based on the number of calls received during their first 12 months of the DM intervention. The number of calls received ranged from zero (primarily due to inaccurate phone numbers) to four calls. The A1c testing rate was calculated for each of these cohorts as the number of eligible members (the denominator) who had at least one A1c test during the 12-month period (the numerator), as evidenced by administrative claims data indicating an A1c test. The LDL testing rates for each cohort were similarly calculated. The testing rates were then plotted against the number of calls received. Because the DM programs address both type 1 


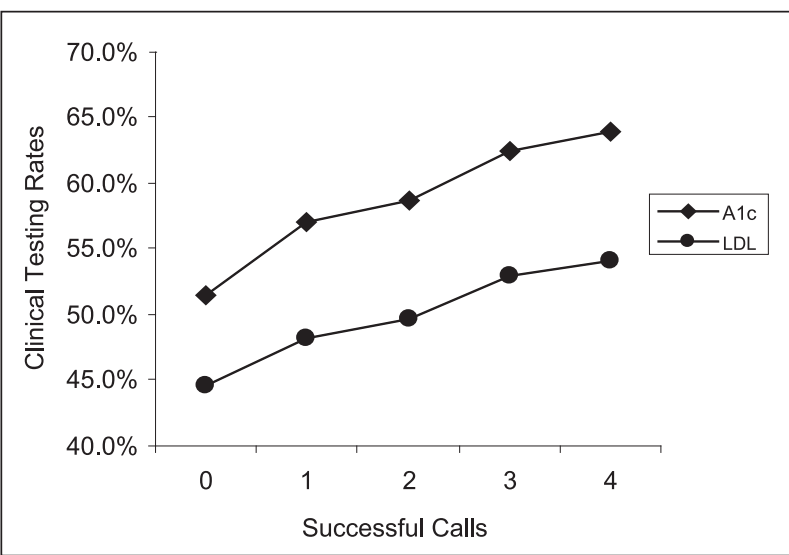

FIG. 1. A1c and LDL testing rates by number of calls.

and type 2 diabetes, these populations were not analyzed separately.

\section{Member characteristics}

Because gender, age, and disease burden are recognized as variables with potential influence on DM outcomes, these were also evaluated as part of the study. Disease burden was estimated based on the amount of healthcare resources a member utilized and expressed as a resource utilization band (RUB) score ranging from zero (no utilization) to five (highest utilization) using the Johns Hopkins ACG tool. ${ }^{18}$ A RUB score was calculated for each member based on healthcare utilization during the first 12 months in a DM program.

\section{RESULTS}

A total of 245,668 members continuously enrolled in diabetes DM programs for a consecutive 12-month period were identified. Members were grouped by their first 12 months in the DM program, irrespective of contract year. The study population was $46.0 \%$ female and $54.0 \%$ male. The average age was 52.6 years. The average RUB score (disease burden) was 3.03. Of the 245,668 members, 137,921 (56.1\%) had at least one A1c test in the first 12 months of participation, and 117,765 (47.9\%) had at least one LDL test.

In general, the greater the number of calls received by members during their first 12 months of DM program participation, the greater the
A1c and LDL testing rates (Fig. 1). Members who received four calls demonstrated a $24.1 \%$ and $21.5 \%$ relative increase in A1c and LDL testing rates, respectively, compared to members who received DM mailings alone (zero call group; Fig. 1). Overall, A1c testing rates were greater than LDL testing rates regardless of the number of calls received.

While the total population demonstrated improvement in both metrics, gender influenced A1c and LDL testing rates. Typically, females who received mail alone were less likely to obtain recommended tests than males who only received DM mailings (Fig. 2). However, females were more responsive than males to the calls. Females who received four calls achieved a $27.7 \%$ and $23.6 \%$ relative increase in $\mathrm{A} 1 \mathrm{c}$ and LDL testing, respectively, compared to females receiving mail alone. This level of increase was in contrast to the respective $21.2 \%$ and $19.9 \%$ relative increase obtained by males who received calls. Therefore, as frequency of calls increased so did testing rates for females, eventually matching or exceeding testing rates obtained by males.

A1c and LDL testing rates also varied by age. Members in the younger group (age 0-19) were more responsive to calls and eventually had markedly higher testing rates than members in other age categories. Younger members called four times achieved a $58.4 \%$ relative increase in A1c testing compared to younger members receiving mail alone (Fig. 3). Of members who received four calls, the 20-64 and 65 or older groups obtained $25.0 \%$ and $33.8 \%$ relative in-

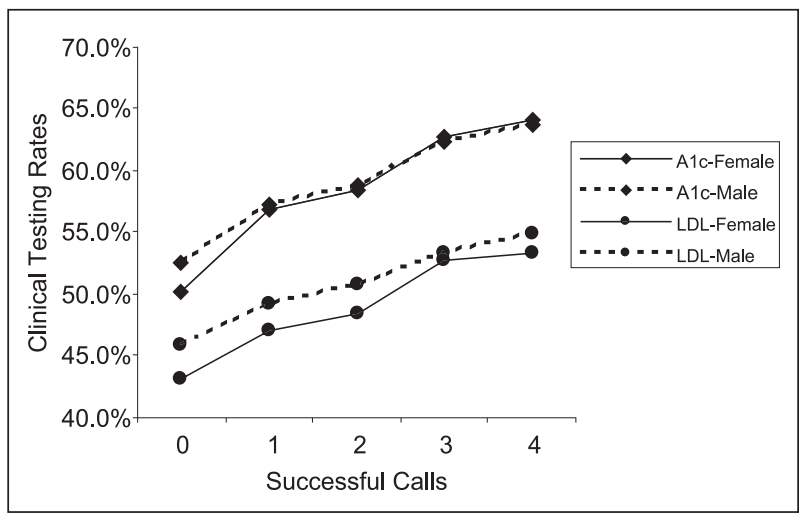

FIG. 2. A1c and LDL testing rates by gender and by call volume. 


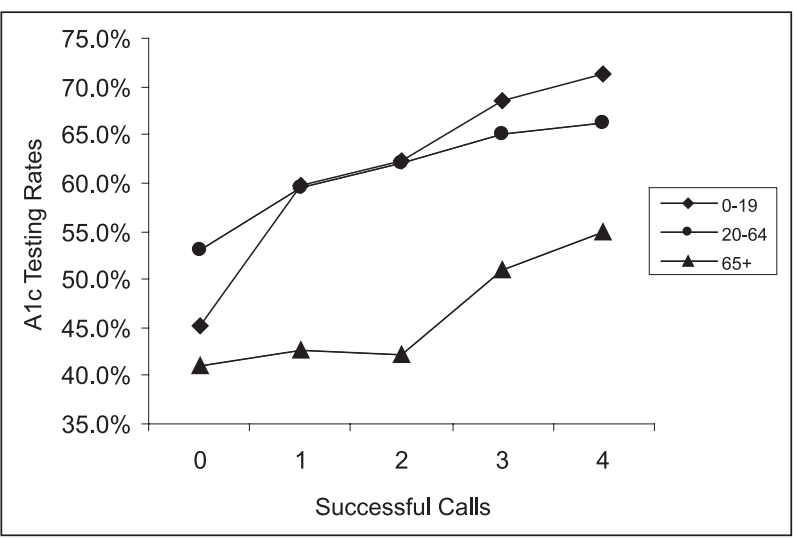

FIG. 3. A1c testing rates by age and call volume.

creases, respectively, in A1c testing compared to the members receiving mail alone.

LDL testing also was influenced by age group, but the effect differed from that observed when examining A1c testing. Members in the 20-64 age group demonstrated the highest LDL testing rates overall. In addition, members aged 20-64 who received four calls achieved a $23.2 \%$ relative increase in LDL testing compared to members receiving mail alone (Fig. 4). While members in the 65 or older age group were less likely to have an LDL test than those in the 20-64 age group, the members in the 65 or older group demonstrated similar levels of improvement. Members in the 65 or older group who received four calls demonstrated a $24.3 \%$ relative increase in testing compared to members receiving mail alone. The youngest members (0-19 years of age) had the lowest LDL testing rate, in sharp contrast to what was observed for A1c testing. However, members ages $0-19$ who received four calls achieved the greatest improvement in LDL testing overall and obtained a $48.1 \%$ relative increase compared to members in this age group who were receiving mail alone.

An association was observed between A1c and LDL testing rates and disease burden, expressed as a RUB score. Members with low disease burden (RUB 0 and RUB 1) had the lowest A1c and LDL testing rate (Fig. 5); however, the members with the lowest disease burden (RUB 0) demonstrated the greatest improvement in A1c and LDL testing in response to calls. Members with the lowest disease burden who received four calls demonstrated 377\% and $488 \%$ improvement in A1c and LDL testing, respectively, compared to members receiving mail alone.

Members with severe disease burden (RUB 4 and RUB 5) had the highest overall testing rates, but achieved more modest improvement in A1c and LDL testing (Fig. 6). High disease burden members with diabetes who received four calls obtained a $12.2 \%$ and $11.5 \%$ relative increase in A1c and LDL testing compared to members receiving mail alone.

\section{DISCUSSION}

This study represents a large-scale and useful approach to understanding DM program outcomes and how these outcomes may vary based on population diversity. There are several distinguishing features of the approach adopted in this study. One key feature was the examination of outcomes and patterns in a uniquely large diabetes DM population that included members from multiple and geographically diverse health plans. This methodology allowed for a more generalizable interpretation of results across various health plans with different benefit structures and employer types. An additional feature was the grouping of members into cohorts based on their first 12 months of program participation, irrespective of contract or calendar year. By grouping members together in this manner, the influence of trends in clinical testing occurring in a given calendar year was minimized. The result was the ability to examine improvement in A1c and

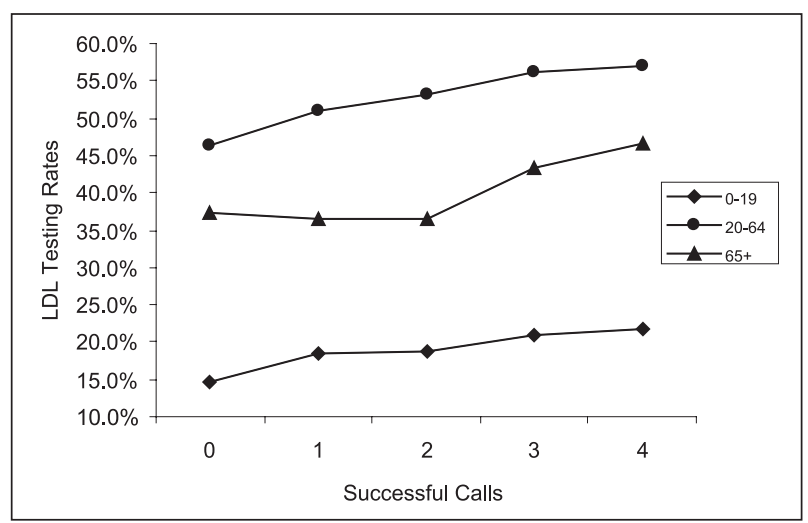

FIG. 4. LDL testing rates by age and by call volume. 


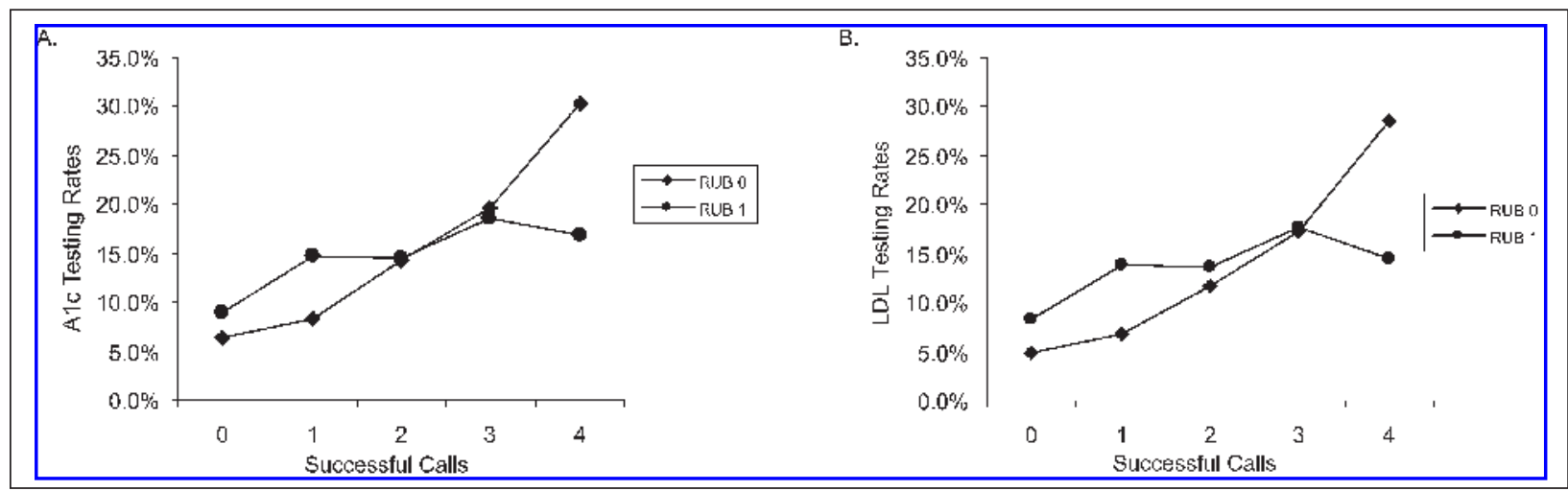

FIG. 5. A1c and LDL testing rates by lower disease burden (RUB) and by call volume.

LDL testing achieved by members participating in DM programs, while minimizing the impact of individual health plan variability or annual healthcare trends.

Analysis of this large diabetes DM program population demonstrated increases in A1c and LDL testing in the first 12 months of participation in the program. A dose-response effect was observed between volume of calls and A1c or LDL testing. Specifically, the call volume was positively associated with increases in testing. A member's outlook or receptiveness to DM may have influenced this relationship. For example, members who received or accepted more calls may have been more motivated toward healthy behaviors than members who received or accepted fewer calls. Future studies will evaluate members' characteristics to assess whether factors potentially influencing adherence to clinical testing can be identified and measured.

In general, A1c testing rates were higher than
LDL testing rates. Age was one variable that influenced responsiveness to the DM program. Specifically, the A1c testing rates obtained by members in the 20-64 and 65 or older age groups differed by $3.6 \%-9.2 \%$ compared to LDL testing rates. This may indicate a broader awareness and acceptance of A1c as the previous gold standard test for diabetes monitoring. The more recently added guideline for LDL testing does not appear to have achieved such standing, despite its importance as part of standards of care for diabetes. ${ }^{8,10}$ Most striking was the difference in A1c and LDL testing rates for the youngest member group (ages 0-19). The A1c testing rates obtained by members in the 0-19 age group differed within call cohorts by $30.3 \%-49.5 \%$ compared to LDL testing rates obtained by members in this same age group. Paradoxically, while the youngest members demonstrated the highest overall A1c testing rates and the greatest improvement in A1c testing compared to the other age groups, they also

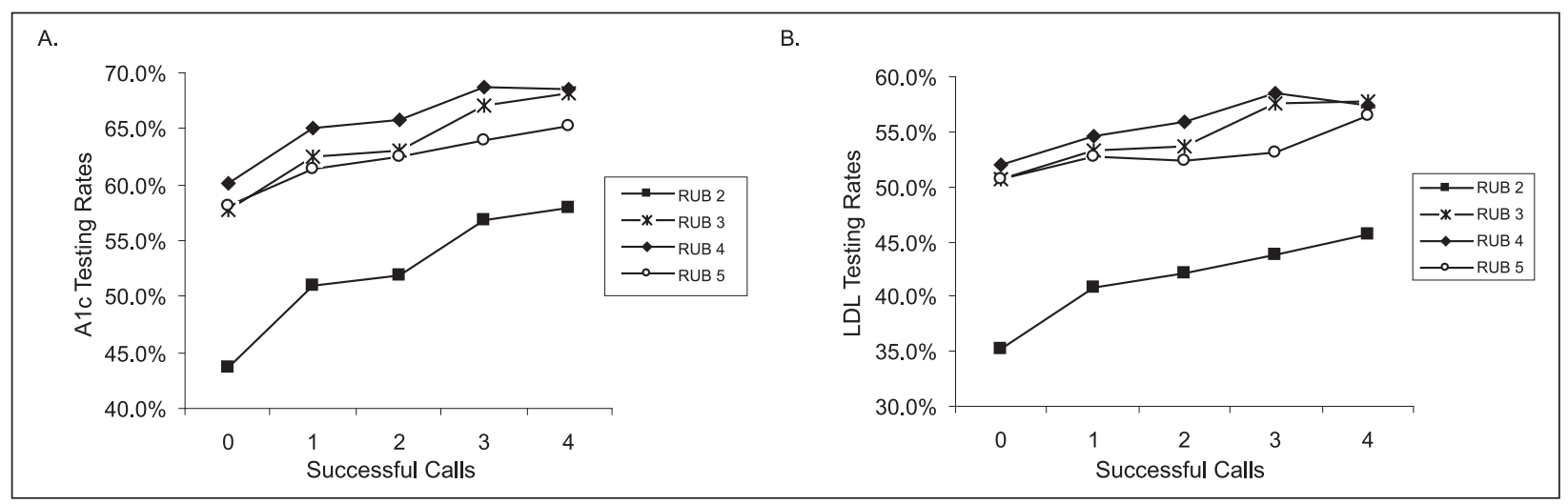

FIG. 6. A1c and LDL testing rates by higher disease burden (RUB) and by call volume. 
had the lowest LDL testing rate compared to the other age groups. This is of concern because hyperlipidemia, specifically elevated LDL levels, is common in children and adolescents with type 1 diabetes, and children and adolescents with elevated LDL levels have been reported to have significantly higher A1c levels. ${ }^{19}$ The results of this study suggest that additional efforts be made to address the importance of LDL testing in general, and that emphasis should be placed on communication with the youngest age groups or their parents.

The potential of DM programs to assist in improving adherence to clinical testing for elderly populations is promising. The quality of care received by elderly Medicare patients with diabetes is suboptimal; beneficiaries are less likely to obtain A1c testing. ${ }^{20,21}$ Recently, federal government-sponsored awards have been received by several DM companies to implement programs that foster adherence to standards of care for Medicare patients. The improvements in A1c and LDL testing reported herein suggest that such a diabetes DM program can benefit the 65 and older population.

Gender was another variable that influenced responsiveness to the DM programs. Females who received mail alone had lower testing rates compared to males who received mail only. This observation is consistent with previous studies where females were found to be less likely to obtain an LDL test and have appropriate lipid management compared to males. ${ }^{22,23}$ For females participating in the diabetes DM programs, it was only after receiving telephonic intervention that the gender differences in A1c and LDL testing rates were reduced. Such findings suggest that the telephonic component of a DM program may be the key to overcoming other potential health disparities experienced by women.

A1c and LDL testing rates varied with disease burden. Members with the greatest level of disease burden (ie, those with the highest RUB score) achieved the highest A1c and LDL testing rates. This is noteworthy because improvement in healthy behaviors (eg, obtaining appropriate clinical tests) is likely to result in better control of the disease state. Interestingly, those with the lowest RUB had the greatest percentage improvement in testing rates. Motivat- ing positive behavior change in members with less severe disease has great potential for preventing or delaying the diabetes complications that are costly in terms of dollars and quality of life.

\section{Limitations}

Limitations of this study include using metrics that are less comprehensive (one or more tests per year) than those suggested by national guidelines and less stringent than those included in the NCQA's Health Plan Employer Data and Information Set (HEDIS) Comprehensive Diabetes Care (CDC) measure (eg, two or more A1c and LDL tests per year). The purpose of the HEDIS CDC measure is to evaluate the performance of health plans in fostering member adherence to standards, whereas this study focused on estimating the power of a DM telephonic intervention to stimulate positive behavior change in a large, heterogeneous diabetes population. Receiving just one A1c test in this study is a proxy for the initiation of positive healthy behavior change.

An additional difference is that the HEDIS CDC measure of adherence to clinical testing can be calculated based on the combination of administrative claims and medical records (ie, chart audit) data. ${ }^{24}$ HEDIS CDC measures of adherence are able to use medical records because they can evaluate smaller random samples of larger populations for their calculations. In contrast, this study was more conservative and used only administrative claims data but focused on a much larger population. As a result, it is likely that the testing rates reported herein are conservative estimates and the actual rates are likely to be uniformly higher. Medical records can be a better source than administrative claims data for measuring clinical testing, and the combination of the two sources provides an even more complete accounting. ${ }^{25}$ Evaluating medical records for performance of clinical tests is often cost prohibitive, and especially so given the large sample size of this particular study population.

\section{Indications for further study}

This study addressed several important questions regarding the effect of telephonic DM 
interventions on members' healthcare behavior. One of the most valuable results demonstrated the relationship between the volume of calls and the desired behavior change in a large, diverse diabetes population from multiple national health plans. This study also provided insights into the differential responses of members to telephonic intervention based on age, gender, and disease burden.

The results also suggested indications for further exploration, including the following:

- Statistical models representing the relationship between the frequency of telephonic intervention and adherence rates (ie, based on the observed dose-response relationship).

- A targeted, prospective clinical evaluation of whether the observed differences in age, gender, and disease burden can guide improvement of intervention strategies and whether specialized interventions for each demographic combination would further enhance DM program effectiveness.

- An analysis to determine whether increases in process measures (ie, obtaining a test) correlates with improvements in the corresponding clinical measures (ie, laboratory values).

- Further evaluation of how process measures and clinical measures are correlated with cost savings.

\section{CONCLUSION}

This study demonstrates that telephonic intervention, as part of Healthways DM programs, can successfully motivate members with diabetes to improve their adherence to recommended clinical testing. Because this study was conducted with a large, diverse diabetes population, these findings will likely have broad applicability across other commercial health plans. The observation that testing is influenced by gender, age, and disease burden is of value in designing programs that target specific populations such as those in employer-funded health plans or Medicare health plans. Such knowledge gives DM providers necessary strategies for helping members mod- ify their behaviors and improve their overall quality of care.

\section{REFERENCES}

1. Hogan P, Dall T, Nikolov P, American Diabetes Association. Economic costs of diabetes in the U.S. in 2002. Diabetes Care 2003;26:917-932.

2. The effect of intensive treatment of diabetes on the development and progression of long-term complications in insulin-dependent diabetes mellitus. The Diabetes Control and Complication Trial Research Group. N Engl J Med 1993;329:977-986.

3. Wagner EH, Sandhu M, Newton KM, McCulloch DK, Ramsey SD, Grothaus LC. Effect of improved glycemic control on health care costs and utilization. JAMA 2001;285:182-189.

4. Vijan S, Hofer TP, Hayward RA. Estimated benefits of glycemic control in microvascular complications in type 2 diabetes. Ann Intern Med 1997;127:788-795.

5. Robertson KE, Prince MJ. What the UKPDS really says about cardiovascular disease and glycemic control. Clin Diabetes 1999;17:109-114.

6. Grundy SM, Cleeman JI, Merz CN, et al. Implications of recent clinical trials for the National Cholesterol Education Program Adult Treatment Panel III Guidelines. J Am Coll Cardiol 2004;44:720-732.

7. Turner RC, Millns H, Neil HA, et al. Risk factors for coronary artery disease in non-insulin dependent diabetes mellitys (UKPDS 23). BMJ 1998;316:823-828.

8. Haffner SM, American Diabetes Association. Dyslipidemia management in adults with diabetes. Diabetes Care 2004;27:S68-S71.

9. National Institute of Diabetes and Digestive and Kidney Diseases (NIDDK). National diabetes statistics. Available at: www.diabetes.niddk.nih.gov/dm/ pubs/statistics/index.htm. Accessed February 15, 2007.

10. American Diabetes Association: Standards of medical care in diabetes-2006. Diabetes Care 2006;29:S4-S42.

11. Delamater AM. Clinical use of hemoglobin $\mathrm{A}_{1 \mathrm{C}}$ to improve diabetes management. Clin Diabetes 2006; 24:6-8.

12. Heisler M, Piette JD, Spencer M, Kieffer E, Vijan S. The relationship between knowledge of recent $\mathrm{HbA}_{1 \mathrm{C}}$ values and diabetes care understanding and selfmanagement. Diabetes Care 2005;28:816-822.

13. Larsen ML, Horder M, Mogensen EF. Effect of longterm monitoring of glycosylated hemoglobin levels in insulin-dependent diabetes mellitus. $\underline{N \text { Engl J Med }}$ 1990;323:1021-1025.

14. Orr PM, McGinnis MA, Hudson LR, et al. A focused telephonic nursing intervention delivers improved adherence to A1c testing. Dis Manag 2006;9:277-283.

15. Snyder JW, Malaskovitz J, Griego J, et al. Quality improvement and cost reduction realized by a purchaser through diabetes disease management. Dis Manag 2003;6:233-241. 
16. Steffen B. Cost effective management of type 2 diabetes: providing quality care in a cost-constrained environment. Am J Manag Care 2000;6:S697-S703.

17. Summary evidence table: disease management. Available at: www.thecommunityguide.org/diabetes/dmdisease-mgt-tables.pdf. Accessed February 15, 2007.

18. Carlsson L, Borjesson U, Edgren L. Patient-based "burden-of-illness" in Swedish primary health care. Applying the Johns Hopkins ACG case-mix system in a retrospective study of electronic patient records. Int J Health Plann Manag 2002;17:269-282.

19. Wiltshire EJ, Hirte C, Couper JJ. Dietary fats do not contribute to hyperlipidemia in children and adolescents with type 1 diabetes. Diabetes Care 2003;26: 1356-1361.

20. McCall DT, Sauaia A, Hamman RF, et al. Are low-income elderly patients at risk for poor diabetes care? Diabetes Care 2004;27:1060-1065.

21. Pham HH, Schrag D, Hargraves JL, et al. Delivery of preventative services to older adults by primary care physicians. IAMA 2005;214:473-481.

22. Kim C, Kerr EA, Bernstein SJ, et al. Gender disparities in lipid management: the presence of disparities depends on the quality measure. Am J Manag Care 2006;12:133-136.

23. Nau DP, Mallya U. Sex disparity in the management of dyslipidemia among patients with type 2 diabetes mellitus in a managed care organization. Am J Manag Care 2005;11:69-73.

24. National Committee for Quality Assurance. HEDIS 2006 technical specifications. Comprehensive Diabetes Care 2006;2:124-133.

25. Keating NL, Landrum MB, Landon BE, et al. Measuring the quality of diabetes care using administrative data: is there bias? Health Serv Res 2003;38: 1529-1545.

Address reprint requests to: Carter R. Coberley, Ph.D. Healthways, Inc.

3841 Green Hills Village Dr., Ste. 300 Nashoille, TN 37215

E-mail: Carter.Coberley@healthways.com 